\title{
ALTERNATING PROCEDURES IN UNIFORMLY SMOOTH BANACH SPACES
}

\author{
I. ASSANI
}

(Communicated by John B. Conway)

\begin{abstract}
Let $E$ be a uniformly smooth Banach space and $C$ the set of real continuous strictly increasing functions $\mu$ on $\mathbf{R}_{+}$such that $\mu(0)=0$. At each $\mu$ we can associate a unique duality $\operatorname{map} J_{\mu}: E \rightarrow E^{*}$ such that $\left(J_{\mu} x, x\right)=$ $\left\|J_{\mu} x\right\| \cdot\|x\|$ and $\left\|J_{\mu} x\right\|=\mu(\|x\|)$. We prove in this note that if $T_{n}$ is a sequence of linear contractions on $E$ the sequence $T_{1}^{*} T_{2}^{*} \cdots T_{n}^{*} J_{\mu} T_{n} \cdots T_{2} T_{1} x$ converges strongly in $E^{*}$ norm for all $x$ in $E$. In particular if $E^{*}$ is also uniformly smooth then for any $\mu$ and $\nu$ in $C$ the sequence $J_{\nu}^{*} T_{1}^{*} T_{2}^{*} \cdots T_{n}^{*} J_{\mu} T_{n} \cdots T_{1} x$ converges in $E$ norm. This generalizes a result of M. Akcoglu and L. Sucheston [1].
\end{abstract}

Introduction. Let $E$ be a uniformly smooth Banach space, i.e., $\lim _{t \rightarrow 0}((\| x+$ $t h\|-\| x \|) / t)$ exists uniformly in $x,(x \neq 0)$ and $h$. It is known that the dual $E^{*}$ of $E$ is then uniformly convex.

So for $\varepsilon>0$ there exists $\delta>0$ such that if $\|x+y\|>2-\delta,\|x\| \leq 1$, and $\|\mid y\| \leq 1$, then $\|x-y\|<\varepsilon\left(x, y \in E^{*}\right)$. We denote by $C$ the set of real strictly increasing continuous functions verifying $\mu(0)=0$ defined on $[0,+\infty[$. The space $E$ being smooth, there exists a unique duality map $J_{\mu}$ for any $\mu \in C, J_{\mu}: E \rightarrow E^{*}$ satisfying the following conditions

(i) for any $x$ in $E,\left(J_{\mu} x, x\right)=\left\|J_{\mu} x\right\| \cdot\|x\|$;

(ii) for any $x$ in $E,\left\|J_{\mu} x\right\|=\mu(\|x\|)$.

If $E$ is uniformly smooth then $J_{\mu}$ is uniformly continuous on the bounded sets of $E$ (for the norm topology) to $E^{*}$ (with the norm topology). (For a reference on these notions see [4]).

In [5] G. C. Rota introduced the following procedure for $L^{1}$ and $L^{\infty}$ positive contractions. He considered the products $T_{1}^{*} T_{2}^{*} \cdots T_{n}^{*} T_{n} \cdots T_{1}$. We showed in [2] the norm convergence of such products when the operators $T_{i}$ are linear contractions on a Hilbert space. When the Banach space $E$ is not a Hilbert space, $T$ and $T^{*}$ do not act on the same space. It seems then necessary to introduce a duality map $J_{\mu}$ in order to use the product $T_{1}^{*} T_{2}^{*} \cdots T_{n}^{*}$.

Our first result is the norm convergence of the products $T_{1}^{*} T_{2}^{*} \cdots T_{n}^{*} J_{\mu} T_{n} T_{n-1}$ $\cdots T_{1} x$ in $E^{*}$ norm for any duality map and any sequence $T_{i}$ of linear contractions in the uniformly smooth Banach space $E$. Using the fact (see [4]) that there exists an equivalent norm on $E^{*}$ for which $E^{*}$ is uniformly smooth, we can define $J_{\nu}^{*}$ a duality map on $E^{*}$ for any $\nu$ in $C$. We prove then the norm convergence in $E$ norm of the products $J_{\nu}^{*} T_{1}^{*} T_{2}^{*} \cdots T_{n}^{*} J_{\mu} T_{n} \cdots T_{1} x$ for any $x$ in $E$. This extends the result of M. Akcoglu and L. Sucheston [1] proved in $L^{p}$ and for particular duality maps.

Received by the editors February 19, 1988.

1980 Mathematics Subject Classification (1985 Revision). Primary 28D99, 47A35.

Key words and phrases. Uniformly smooth Banach spaces, duality map, pointwise convergence. 
As far as the author knows Rota's pointwise theorem has only been proved for positive operators even if we know in the nonpositive case of $L^{1}, L^{\infty}$ contractions that we have a dominated estimate in $L^{p}(1<p<\infty)$ or $L \log L$ (by considering the linear modulus of each operator). In [3] we study the pointwise convergence for nonpositive operators.

THEOREM 1. Let $E$ be a uniformly smooth Banach space and $T_{n}$ a sequence of (linear) contractions on $E$. For any duality map $J_{\mu}$ and any $x$ in $E$ the sequence $S_{n}(x)=T_{1}^{*} \cdots T_{n}^{*} J_{\mu} T_{n} \cdots T_{1} x$ converges in $E^{*}$ norm.

PROOF. We will use the notations and properties quoted in the introduction. We will prove that $S_{n}(x)$ is a Cauchy sequence. We can assume that $\mu(\|x\|) \leq 1$.

It is easy to see that the sequence $\left\|T_{n} \cdots T_{1} x\right\|$ is decreasing so for any $\varepsilon^{\prime}>0$ there exists $n_{0}$ such that for $n>n_{0}\left(\varepsilon^{\prime}\right)$

$$
\left\|T_{n_{0}} \cdots T_{1} x\right\|-\left\|T_{n} \cdots T_{n_{0}} \cdots T_{1} x\right\|<\varepsilon^{\prime} .
$$

Let us denote $g=T_{n_{0}} \cdots T_{1} x$ and $U_{n}=T_{n} \cdots T_{n_{0}+1}$ and fix $\varepsilon>0$.

We remark that

(a) $\mu$ being strictly increasing continuous and satisfying $\mu(0)=0$, there is $\lambda_{1}$ such that $\lambda \leq \lambda_{1}$ implies $\mu(\lambda) \leq \varepsilon / 2$.

(b) $E^{*}$ being uniformly convex there exists $\delta>0$ such that $\|x+y\|>2-\delta$ implies $\|x-y\|<\varepsilon$ for $\|x\| \leq 1,\|y\| \leq 1$. We note $\delta^{\prime}=1-\sqrt{1-\delta}$.

(c) $\mu$ is uniformly continuous on $\mu^{-1}([0,1])$. So there exists $\delta^{\prime \prime}$ such that $|\alpha-\beta|<$ $\delta^{\prime \prime}$ implies $|\mu(\alpha)-\mu(\beta)|<\delta^{\prime} \mu\left(\lambda_{1}\right)$.

Let us take $\varepsilon^{\prime}=\operatorname{Min}\left(\lambda_{1}, \delta^{\prime}, \delta^{\prime \prime}\right)$. We claim that $\left\|J_{\mu} g-U_{n}^{*} J_{\mu} U_{n} g\right\|<\varepsilon$. We distinguish two cases.

(i) If $\|g\| \leq \lambda_{1}$ then

$$
\begin{aligned}
\left\|J_{\mu} g-U_{n}^{*} J_{\mu} U_{n} g\right\| & \leq\left\|J_{\mu} g\right\|+\left\|U_{n}^{*} J_{\mu} U_{n} g\right\| \\
& \leq \mu(\|g\|)+\mu\left(\left\|U_{n} g\right\|\right) \\
& \leq 2 \mu(\|g\|) \leq 2 \mu\left(\lambda_{1}\right) \leq \varepsilon .
\end{aligned}
$$

(ii) If $\|g\|>\lambda_{1}$ then

$$
\begin{aligned}
\|g\| \cdot\left\|J_{\mu} g+U_{n}^{*} J_{\mu} U_{n} g\right\| & \geq\left(g, J_{\mu} g\right)+\left(g, U_{n}^{*} J_{\mu} U_{n} g\right) \\
& =\|g\| \cdot\left\|J_{\mu} g\right\|+\left(U_{n} g, J_{\mu} U_{n} g\right) \\
& =\|g\| \cdot\left\|J_{\mu} g\right\|+\left\|U_{n} g\right\| \cdot\left\|J_{\mu} U_{n} g\right\| \\
& =\|g\| \cdot \mu(\|g\|)+\left\|U_{n} g\right\| \cdot \mu\left(\left\|U_{n} g\right\|\right) .
\end{aligned}
$$

So

$$
\begin{aligned}
\frac{\left\|J_{\mu} g+U_{n}^{*} J_{\mu} U_{n} g\right\|}{\mu(\|g\|)} & \geq 1+\left(1-\frac{\varepsilon^{\prime}}{\|g\|}\right) \cdot \frac{\mu\left(\|g\|-\varepsilon^{\prime}\right)}{\mu(\|g\|)} \\
& \geq 1+\left(1-\frac{\varepsilon^{\prime}}{\lambda_{1}}\right) \cdot\left(1-\frac{\delta^{\prime} \mu\left(\lambda_{1}\right)}{\mu(\|g\|)}\right) \\
& \geq 1+\left(1-\delta^{\prime}\right)\left(1-\delta^{\prime}\right) \\
& \geq 2-\delta .
\end{aligned}
$$

As

$$
\frac{\left\|J_{\mu} g\right\|}{\mu(\|g\|)}=\frac{\mu(\|g\|)}{\mu(\|g\|)}=1
$$


and

$$
\frac{\left\|U_{n}^{*} J_{\mu} U_{n} g\right\|}{\mu(\|g\|)} \leq \frac{\left\|J_{\mu} U_{n} g\right\|}{\mu(\|g\|)}=\frac{\mu\left(\left\|U_{n} g\right\|\right)}{\mu(\|g\|)} \leq \frac{\mu(\|g\|)}{\mu(\|g\|)} \leq 1
$$

( $U_{n}^{*}$ being a contraction), hence by the uniform convexity of $E^{*}$ we have that

$$
\left\|J_{\mu} g-U_{n}^{*} J_{\mu} U_{n} g\right\|<\varepsilon \mu(\|g\|) \leq \varepsilon \mu(\|x\|) \leq \varepsilon .
$$

Now to prove Theorem 1 we have just to multiply by $T_{1}^{*} T_{2}^{*} \cdots T_{n}^{*}$ (which is a contraction) to get

$$
\left\|T_{1}^{*} \cdots T_{n_{0}}^{*} J_{\mu} T_{n_{0}} \cdots T_{1} x-T_{1}^{*} \cdots T_{n}^{*} J_{\mu} T_{n} \cdots T_{1} x\right\|<\varepsilon
$$

for $n \geq n_{0}(\varepsilon)$.

COROLLARY 2. Let $E$ be a uniformly smooth Banach space and $T_{n}$ a sequence of linear contractions on $E$. For $\nu$ in $C$ and $J_{\nu}^{*}: E^{*} \rightarrow E$ any duality map (for an equivalent norm on $E^{*}$ making $E^{*}$ uniformly smooth) the sequence

$$
J_{\nu}^{*} T_{1}^{*} T_{2}^{*} \cdots T_{n}^{*} J_{\mu} T_{n} \cdots T_{1} x
$$

converges in $E$ norm for all $x$ in $E$.

PROOF. Now by Theorem 1 the sequence $T_{1}^{*} \cdots T_{n}^{*} J_{\mu} T_{n} \cdots T_{1} x$ is a Cauchy sequence in $E^{*}$ for the initial norm \|\|$_{E}$.

By P. Enflo's result (see [4]) there exists an equivalent norm $\|||||_{E^{*}}$ on $E^{*}$ making $E^{*}$ uniformly smooth. So for any $\nu \in C$ there exists a duality map $J_{\nu}^{*}: E_{\|\|}^{*}\|\|_{E^{*}} \rightarrow$ $E_{\|\|}\|\|_{E}$ which is uniformly continuous on the bounded sets of $E^{*}$. The norm \|\|$\|_{E^{*}}$ being equivalent to the norm \|\|$_{E^{*}}$, the norm \|\|\|\|$_{E}$ it induces on $E$ is also equivalent to \|\|$_{E}$.

Hence if the sequence $x_{n}$ is a Cauchy sequence for \|\|$_{E^{*}}$ it is also a Cauchy sequence for \|\|\|\|$_{E^{*}}$. By the continuity of $J_{\nu}^{*}$ the sequence $J_{\nu}^{*} x_{n}$ is then a Cauchy sequence in $E_{\|\|} \|_{E}$ and so a Cauchy sequence for $E_{\|\|_{E}}$. This implies that the sequence $\left(J_{\nu}^{*} x_{n}\right)$ converges in $E$ for the norm \|\|$_{E}$.

REMARK. After the first version of this paper was completed we obtained in [3] the pointwise convergence in $L \log L$ for nonpositive $L^{1}-L^{\infty}$ contractions.

\section{REFERENCES}

1. M. A. Akcoglu and L. Sucheston, An alternating procedure for operators on $L^{p}$ spaces, Proc. Amer. Math. Soc. 99 (1987), 555-558.

2. I. Assani, Pointwise convergence of sequence of operators on $L_{E}^{p}$ spaces, (preprint).

3. __ Rota's alternating procedure with nonpositive operators, (preprint).

4. J. Diestel, Geometry of Banach spaces-selectred topics, Lecture Notes in Math., vol. 485, Springer-Verlag.

5. G. C. Rota, An "alternierende Verfahren" for general positive operators, Bull. Amer. Math. Soc. 68 (1962), 95-102.

Department of Mathematics, University of TORONTO, TORONTO, Ontario, CANADA M5S 1A1 